\title{
APORTES DE LA COMUNICACIÓN PARA LA DIFUSIÓN DEL PATRIMONIO CULTURAL
}

\section{COMMUNICATION CONTRIBUTIONS FOR THE DISSEMINATION OF CULTURAL HERITAGE.}

\author{
María Walls Ramírez¹. Universidad Pedagógica Experimental Libertador. \\ Venezuela. \\ mfwr26@gmail.com
}

\section{RESUMEN}

El artículo pretende aportar algunas reflexiones sobre la estrecha relación existente entre la cultura y la comunicación, como reflejo de la sociedad, de la creación y el quehacer humano. El estudio de estas dos dimensiones es de vital importancia en la difusión y divulgación del patrimonio cultural. Mediante una revisión bibliográfica se extrajeron las principales nociones, aspectos y características más resaltantes que identifican las áreas de la comunicación y gestión cultural, abordado desde la perspectiva de algunos investigadores y especialista en la materia.

PALABRAS CLAVE: cultura; comunicación; patrimonio; difusión; espacios culturales; estrategias.

\section{ABSTRACT}

The article aims to provide some reflections on the close relationship between culture and communication, as a reflection of society, of creation and human activity. The study of these two dimensions is of vital importance in the dissemination and dissemination of cultural heritage. Through a bibliographic review, the main notions, aspects and most outstanding characteristics that identify the areas of communication and cultural management were extracted, approached from the perspective of some researchers and specialists in the field.

KEYWORDS: culture; communication; heritage; dissemination; cultural spaces; strategies.

\section{Cómo citar el artículo:}

Walls Ramírez, M. (2020). Aportes de la comunicación para la difusión del patrimonio cultural. Revista de Ciencias de la Comunicación e Información, 25(1), 49-55

doi: http://doi.org/10.35742/rcci.2020.25(1).49-55

\footnotetext{
${ }^{1}$ María Walls Ramírez: docente de la Universidad Pedagógica Experimental Libertador. 
El uso adecuado de la terminología que encierra la difusión del patrimonio es necesario para aplicar estrategias de comunicación efectivas y poder comprender el significado y trascendencia de la comunicación en la vida y devenir de la sociedad, de las generaciones pasadas, presentes y futuras, las cuales han tenido, tienen y tendrán sus propios códigos de comunicación. Pasquali (1990, p. 63) presenta una clara distinción entre información (referida a la alocución de mensajes), comunicación (relacionado diálogo) y conocimiento (que lo ubica dentro de la difusión)

De lo anterior, se concluye que estás tres palabras se interrelacionan entre sí creándose la siguiente trilogía: se difunde una información usando canales y medios de comunicación para que sea del conocimiento público o privado.

Desde la visión antropológica-cultural, la comunicación, como forma de expresión humana-social es esencial en la transmisión de los saberes ancestrales. Comunicar es trasferir conocimiento y en el caso del patrimonio cultural al promocionar y divulgar manifestaciones culturales es un acto comunicacional en sí mismo.

Para Pantin (2009) la cultura puede concebirse como:

un sistema de organización, producción y relaciones sociales interconectadas por la acción comunicativa de ideas, creencias y valores que definen o caracterizan a una época y un grupo social determinado, englobando todo el conjunto de representaciones...que son transmitidos de generación en generación como códigos simbólicos que constituyen la identidad de un pueblo y facilitan la comunicación entre los miembros del grupo que la comparten. ( $p$. 393).

La cultura como sistema de interacciones hombre-sociedad, incluye a la comunicación como instrumento de cohesión o división, de encuentro o confrontación. Pasquali (1990), plantea que "La Cultura y Comunicación son el anverso y el reverso de un mismo proceso...todo cambio de sistema comunicacional es un cambio de la estructura socio-cultural" (p. 163).

Así pues, los términos tales como: hegemonía comunicacional, cultura de masas, democratización de la información, diálogo entre culturas, entre otros; centran su atención en la estrecha relación entre cultura y comunicación.

El patrimonio cultural según la UNESCO es "la herencia cultural del pasado propia de una comunidad, con la que vivimos en la actualidad y que se trasmiten a las generaciones presentes y futuras. Una fuente insustituible de vida e inspiración". (Arrieta, 2018, p. 12). Se entiende entonces que, la cultura es transmitida a través de la tradición oral o escrita quedando como legado para las generaciones futuras.

Pasquali (1990, p. 168) destaca el rol que desempeñan los medios de comunicación de masas en la transmisión de "valores abstractos, informales e interferibles" la "alienación de las culturas" desde lo "abstracto a lo concreto" y su influencia en la cultura nacional.

El acto de comunicar en sí mismo forma parte de la cultura, por el uso de simbolismos y significados que dan cuerpo e identidad cultural propia. Los códigos 


\section{APORTES DE LA COMUNICACIÓN PARA LA DIFUSIÓN DEL PATRIMONIO CULTURAL}

lingüísticos usados en la tradición oral y escrita, así como, los sonidos y la imagen como códigos utilizados en el lenguaje audiovisual son medios de transmisión para la difusión del patrimonio cultural.

Por su parte, Arrieta (2018) los saberes, tradiciones y creencias son definidos como: el conjunto de conocimientos y modos de hacer enraizados en la vida cotidiana de las comunidades. Formas de ser y de pensar que se han transmitido oralmente o a través de un proceso de recreación colectiva, desde actividades concretas comunitarias hasta leyendas, dichos, historias y creencias (p. 15).

Con respecto a la relación simbiótica entre comunicación y cultura Monge (2017) destaca:

Valor simbólico/comunicativo: que entiende que los objetos históricos simbolizan algo perteneciente al pasado (un hecho, una idea, etc.) y por lo tanto tienen la cualidad de portar un mensaje. De esta forma, pasan a ser signos que recuerdan algo pasado $\mathrm{y}$, por tanto, tienen un alto valor comunicativo. (p. 13)

Para poder comunicar el patrimonio cultural y transmitir su valor y significado, se debe tener claros las nociones de divulgación y difusión. Para este autor, divulgar el patrimonio significa:

Dar a conocer al público los contenidos culturales que se asocian a los recursos patrimoniales; procurando establecer una relación comunicativa eficaz ente el bien cultural y el público. Es decir, obteniendo una recepción y entendimiento correcto de lo que queremos transmitir. (p. 18).

Por otro lado, la difusión se concibe como “...una estrategia comunicativa que consiste en la construcción de discursos generados alrededor de los recursos patrimoniales, para favorecer la conexión comunicativa con el público al que se dirigen (Monge, 2017 p. 17).

Tal es el caso del vertiginoso desarrollo, en la era de la información del sector cuaternario: las personas se comunican en vivo y directo mediante redes de comunicación como la expresión de un mundo polarizado. El tiempo y la distancia ya no son una limitante sino una oportunidad.

Güerere (1999, p. 87), ya pronosticaba el avance de este sector en la economía mundial "En la actualidad estas industrias cuaternarias tienen un alto impacto sobre la generación de empleo y la generación de riquezas para los países" y se refería solamente a la industria del arte y del entretenimiento: cine, medios audiovisuales y las telecomunicaciones.

La UNESCO define las industrias culturales como todas aquellas actividades que se desarrollan con el propósito de producir o reproducir bienes, servicios o actividades de "contenido cultural, artístico o patrimonial" (Arrieta, 2018) mediante su promoción, difusión o comercialización.

El surgimiento y auge de las industrias culturales como expresión de la globalización, permiten la difusión y promoción del turismo cultural, invitan a los 
espectadores a vivir la experiencia de interactuar (de forma física o digital) con otras comunidades, personas, tradiciones y costumbres, pasando de un ámbito local a uno internacional, por medio de aplicaciones móviles, CD y programas interactivos, páginas web, cursos, congresos y seminarios en línea, canales de videos, videojuegos, avatares, recorridos virtuales a museos y bibliotecas, entre otros.

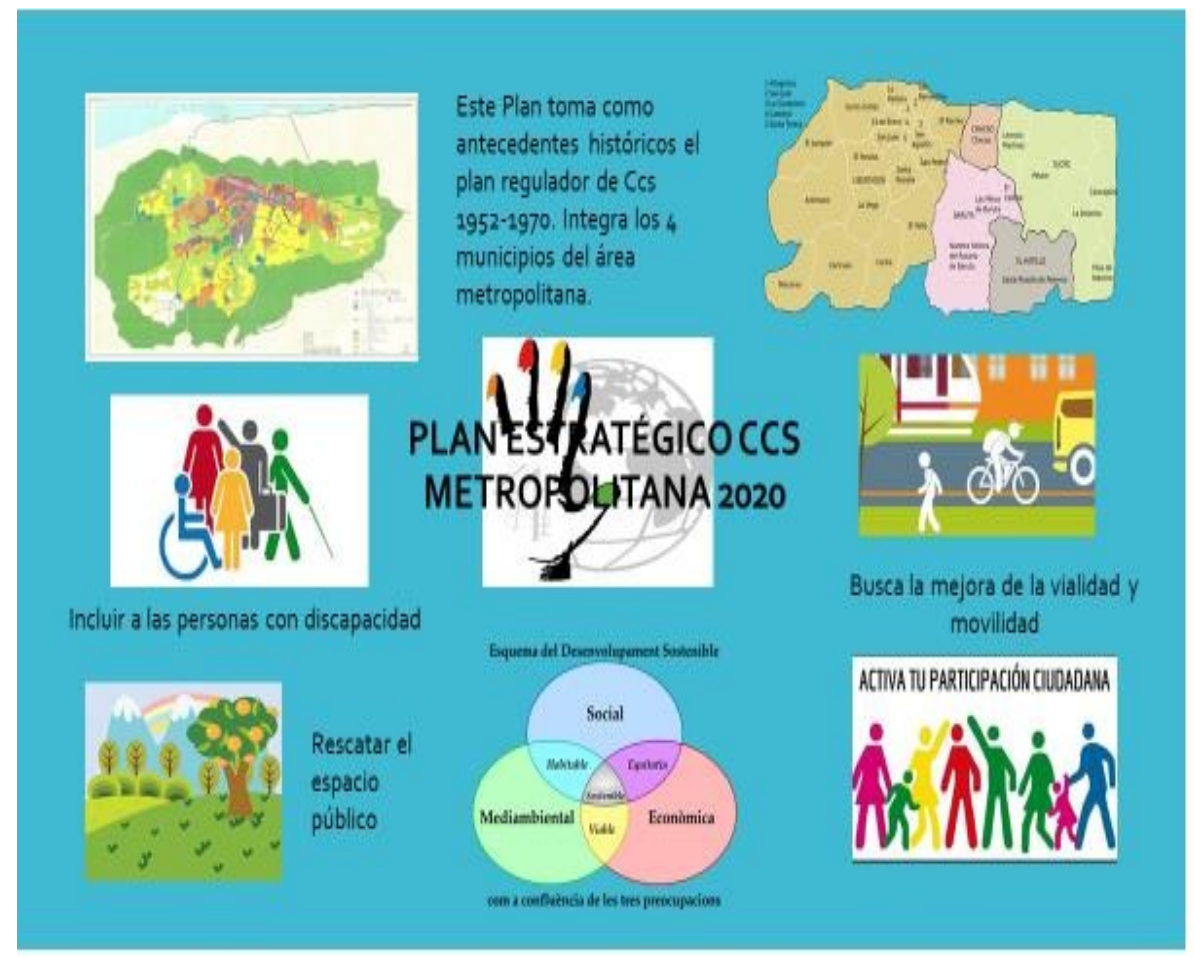

Para lograr que una ciudad sea
sostenible debe existir: Inclusión,
participación,
Desarrollar actividades
productivas de inversión de capital
humano, monetario y tecnológico
garantizando el menor impacto
ambiental posible. Promover la
creatividad a través del fomento
de las industrias culturales
creativas. Apoyar los
emprendimientos individuales y
colectivos.
El éxito de una sociedad
educadora está en la formación
continua de sus ciudadanos
responsables, comprometidos,
innovadores, tolerantes.
EDUCACióN = cambio en el
comportamiento social
significativo que trascienda
generaciones.
Hay que reconciliarnos con
Caracas.

Fuente: elaboración propia.

A través del esquema anterior, se trata de explicar cómo las estrategias comunicacionales son fundamentales para la promoción y difusión de las políticas públicas referidas a la gestión pública del patrimonio cultural. El plan estratégico 2020 presentado por la Alcaldía de Caracas para el período 2008-2012 tuvo que ser promocionado y dado a conocer a través de estrategias comunicacionales para hacerlo llegar a los entes públicos, privados y la misma ciudadanía de los 5 municipios: conferencias, foros y encuentros donde participaron expertos y miembros de las comunidades que hacen vida en cada entidad municipal, publicaciones, entrevistas en medios de comunicación social, redes sociales y demás recursos web para ese momento.

Los teatros, museos, casas culturales, centros culturales, fundaciones, bibliotecas, galerías y ateneos son espacios promotores de la cultura porque brindan al público diversos servicios: acceso a material bibliográfico y audiovisual para consultas, visitas guiadas, talleres, ofreciendo oportunidades a los artistas y cultores para que muestren sus creaciones en exposiciones.

La relación que se establece entre el museo y la comunicación, por ejemplo, es estrecha. De acuerdo con el Consejo Internacional de los Museos (ICOM) una de las principales funciones de todo museo es la de comunicar, pero "para que esta función 


\section{APORTES DE LA COMUNICACIÓN PARA LA DIFUSIÓN DEL PATRIMONIO CULTURAL}

de comunicación se desarrolle, debe producirse un acercamiento del museo a la sociedad" (Pérez, 1999, p. 61).

Por tanto, comunicar manifestaciones culturales y artísticas a través de diferentes medios y recursos es una forma de divulgar y dar a conocer el patrimonio cultural.

Para lograr que el patrimonio cultural sea conocido debe ser, según Monge (2017) promocionado:

mediante mensajes efectivos a sus posibles usuarios, con el objetivo de despertar su interés por conocerlo y visitarlo. La decisión que tome un posible visitante dependerá de esta información y de cómo perciba en su imaginario lo que se le ofrece (p. 19).

Por tanto, promover y difundir las manifestaciones artísticas, tradiciones y costumbres de una persona, comunidad, pueblo o nación permite reconocer su legado y trascendencia y permite al espectador-público-visitante apreciar, valorar y preservar el patrimonio cultural.

La vinculación entre las estrategias de comunicación la gestión del patrimonio cultural Pantín (2009) lo destaca:

Las manifestaciones culturales y expresiones artísticas requieren un necesario soporte institucional que apoye su fomento, desarrollo y promoción, a la vez que atienda la oferta y la demanda de sus servicios y facilite su conservación patrimonial, su consolidación como memoria colectiva y disfrute creativo ( $p$. 394)

Por tales razones, la comunicación estará siempre presente en la gestión del patrimonio cultural, como vínculo directo entre el usuario y el prestador de servicio. Esto es posible, a través de estrategias de promoción, difusión y participación (democratización) que permitan que, la cultura sea accesible, llegando a la mayor cantidad de personas posibles y comunidades.

Es aquí, donde los medios de comunicación social y digital son aliados indispensables para los productos e industrias culturales.

De acuerdo con García y Álvarez (2015) "Se necesita comunicar cultura, activar lo que en el público hay de pueblo, que haga posible la experimentación cultural, la experiencia de apropiación y de invención, el movimiento de recreación permanente de su identidad" (p.44).

Toda valoración surge de una interacción directa entre el público con el bien cultural. Un bien cultural no es valioso en sí mismo, dependerá de su apreciación y valoración de quienes lo usen o frecuenten y es aquí donde la comunicación juega un rol fundamental. La cultura es transmitida de generación en generación, a través de la tradición oral o escrita.

Toda obra que forme parte del legado cultural de un pueblo o comunidad y que es considerado patrimonio cultural, debe ser promovido y difundido a través de medios de comunicación para garantizar su conocimiento, conservación, y defensa. 
Por consiguiente, para que un bien cultural sea patrimonio debe darse a conocer para darle el lugar y reconocimiento que se merece y para ello la difusión y promoción es de carácter esencial, obligatorio. Al respecto, García y Álvarez (2015) expresan:

Pensar en estrategias de comunicación relacionadas con mensajes de bien público sobre el patrimonio histórico de una localidad requiere de mayor creatividad y dinamismo, y mediante la planeación estratégica de la comunicación se intenta encontrar un camino hacia un desarrollo patrimonial, organizacional y cultural óptimo, por lo que conformar acciones con objetivos específicos destinadas a promocionar la historia y valores arquitectónicos favorece la preservación y familiarización del público con este. (p. 47).

Toda forma de participación e interacción, usando medios de comunicación, como una visita guiada a un museo a través de un recorrido peatonal (modo tradicional), o virtual (alternativo) genera un sentimiento de pertenencia e identidad, creándose un vínculo afectivo entre ese bien cultural y el público, por su significado por lo que representa, por su simbología o por lo que se recuerda.

En la era digital y del conocimiento, es necesario difundir el patrimonio cultural a través de medios de comunicación tradicionales y alternativos que permitan mostrar al público en general, al ciudadano común y no solo a la comunidad académica, la riqueza cultural de las diferentes expresiones artísticas.

En conclusión, el patrimonio cultural representa la identidad de un pueblo y la comunicación es un elemento de encuentro y de intercambio de ideas e información.

\section{REFERENCIAS}

Alcaldía Metropolitana de Caracas (2008). Avances del plan estratégico Caracas metropolitana 2020. Caracas.

Arrieta, M. (2018). La Cultura. Turismo y cultura: oportunidades para el desarrollo local (material no publicado).

García, A. y Álvarez, A. (2015). Estrategias de comunicación para promocionar el patrimonio cultural urbano tangible (inmueble) en el municipio Cumanayagua, en Apuntes Vol. 28 Bogotá: Universidad Javeriana.

Güerere, A. (1999). Las Megatendencias y su impacto comunicacional en América Latina, en Ciudad, Públicos y Consumo Cultural. Caracas: Fundación Polar.

Monge J. R. (2017). Herramientas de difusión del Patrimonio Cultural en España [Trabajo de grado]. Universidad Oberta de Catalunya. Madrid, España.

Pantin, G. (2009). La Territorialidad institucional de la cultura en Venezuela, en Geo Venezuela Vol. 8. Caracas: Fundación Empresas Polar.

Pasquali, A. (1990). Comunicación y cultura de masas. Caracas: Monte Ávila Editores. 
Pasquali, A. (1990). Comprender la comunicación. Caracas: Monte Ávila Editores.

Pérez-Santos, E. (1999). La utilidad de los estudios de visitantes en los museos: áreas de aplicación y desarrollo futuro, en Ciudad, Públicos y Consumo Cultural. Caracas: Fundación Polar.

\section{AUTORA:}

\section{María Walls Ramírez}

Docente de la Universidad Pedagógica Experimental Libertador (UPEL). Profesora en la Especialidad de Geografía e Historia (2003), con Maestría en Enseñanza de la Historia (2008) egresada de la UPEL Pedagógico de Caracas. Miembro del Personal docente del Departamento de Práctica Profesional, coordinadora institucional de Promoción y Difusión de la Investigación y editora de SAPIENS: Revista Universitaria de Investigación adscrito a la Subdirección de Investigación y Postgrado UPEL Pedagógico de Miranda José Manuel Siso Martínez. Área de investigación en patrimonio cultural inmueble edificado moderno. Profesora en la UCV y la UCAB. Caracas, Venezuela.

mfwr26@gmail.com 